\title{
Left Ventricular to Right Atrial Septal Defect Secondary to Blunt Thoracic Trauma Diagnosed by Transesophageal Echocardiography
}

\author{
VANCE E. WILSON,* M.D., MARVIN M. KIRSCH, ${ }^{* *}$ M.D., \\ THOMAS D. STARKEY,** M.D., and WILLIAM F. ARMSTRONG, ${ }^{*}$ M.D. \\ *Cardiology Division, Department of Internal Medicine, and **Thoracic Division, Department of \\ Surgery, The University of Michigan School of Medicine, Ann Arbor, Michigan
}

A case report is presented of a 58-year-old man who developed a new holosystolic murmur 4 months after a high-speed motor vehicle accident. Cardiac catheterization demonstrated a left-to-right shunt at the right atrial level. Intraoperative transesophageal echocardiography (TEE) identified and localized a discrete atrioventricular septal defect associated with a shunt from the left ventricle to the right atrium without tricuspid regurgitation, findings that were confirmed after surgical exploration. TEE is recommended for patients with a heart murmur and a history of blunt cardiac trauma, may permit early diagnosis, and may allow surgical repair in selected patients without pre-operative catheterization. (ECHOCARDIOGRAPHY, Volume 8, May 1991)

atrioventricular septal defect, transesophageal echocardiography, trauma

Cardiac involvement from high-speed motor vehicle accidents probably occurs more often than realized. Cardiac injury has been said to be the most common unsuspected visceral injury responsible for death in accident victims. Nonpenetrating traumatic injuries to the heart have been well described. ${ }^{1}$ Acute cardiac injuries include contusion, transmural myocardial infarction, laceration or rupture of the pericardium, myocardium, cardiac valves, septa, and coronary arteries. Delayed or late effects include rupture of the interventricular septum and ventricular aneurysm. The purpose of this report is to describe a patient who developed a left ventricular to right atrial fistula with a delayed onset following blunt chest trauma.

\section{Case Report}

D.M., a 58-year-old man, was an unrestrained passenger in a high-speed motor ve-

Address for correspondence and reprints: William F. Armstrong, M.D., University Hospital B1 F215-0022, 1500 East Medical Center Drive, Ann Arbor, MI 48109-0022. Fax: 313-936-7641. hicle accident. He sustained multiple injuries including a closed head injury with left cranial nerve III, IV, and VI deficits, and intra-abdominal hemorrhage requiring repair of superficial liver lacerations and a ruptured left hemidiaphragm. Initial cardiac evaluation revealed no murmur. An aortic arch angiogram was performed for a widened mediastinum on chest $X$ ray; there was no aortic transection or dissection noted.

One day postoperatively, he developed a pericardial friction rub and an electrocardiogram showed second-degree atrioventricular block, an incomplete left bundle branch block, and precordial ST-segment elevation suggestive of acute myocardial injury (an electrocardiogram from 2 years earlier was normal). A radionuclide ventriculogram revealed normal right and left ventricular size and function. A twodimensional echocardiogram, limited to parasternal views without Doppler, revealed normal chamber sizes and function without a pericardial effusion. Creatine phosphokinase peaked at 9,900 (normal 30-240 IU/mL) with 2.2\% MB fraction. Eight days after the acci- 
dent, he developed transient atrial fibrillation with a rapid ventricular response. As his pericardial rub resolved, a grade II out of VI systolic ejection murmur was first noted. After a complicated 1-month hospital course, he was discharged home.

Four months after the accident, he was evaluated for dyspnea and fatigue occurring on minimal exertion that had been progressive since hospital discharge. Pulsating neck veins and a grade IV out of VI widely radiating holosystolic murmur were noted. There was mild cardiomegaly on chest $\mathrm{X}$ ray. The electrocardiogram revealed first-degree atrioventricular block and complete left bundle branch block. A two-dimensional echocardiogram with Doppler and color flow imaging revealed mild left and right atrial enlargement, abnormal septal motion consistent with right ventricular volume overload, and a $4.1 \mathrm{M} / \mathrm{sec}$ unusually oriented jet directed into the right atrium, which arose near the base of the septal leaflet of the tricuspid valve.

Five months after the accident, a cardiac catheterization was performed with hemodynamic data (Table I) indicating a left-to-right shunt at the right atrial level. The ratio of pulmonic to systemic flow was 3:1. Left ventriculography revealed passage of contrast medium directly into the right atrium, suggestive of an atrioventricular septal defect, although a membranous ventricular septal defect with tricus-

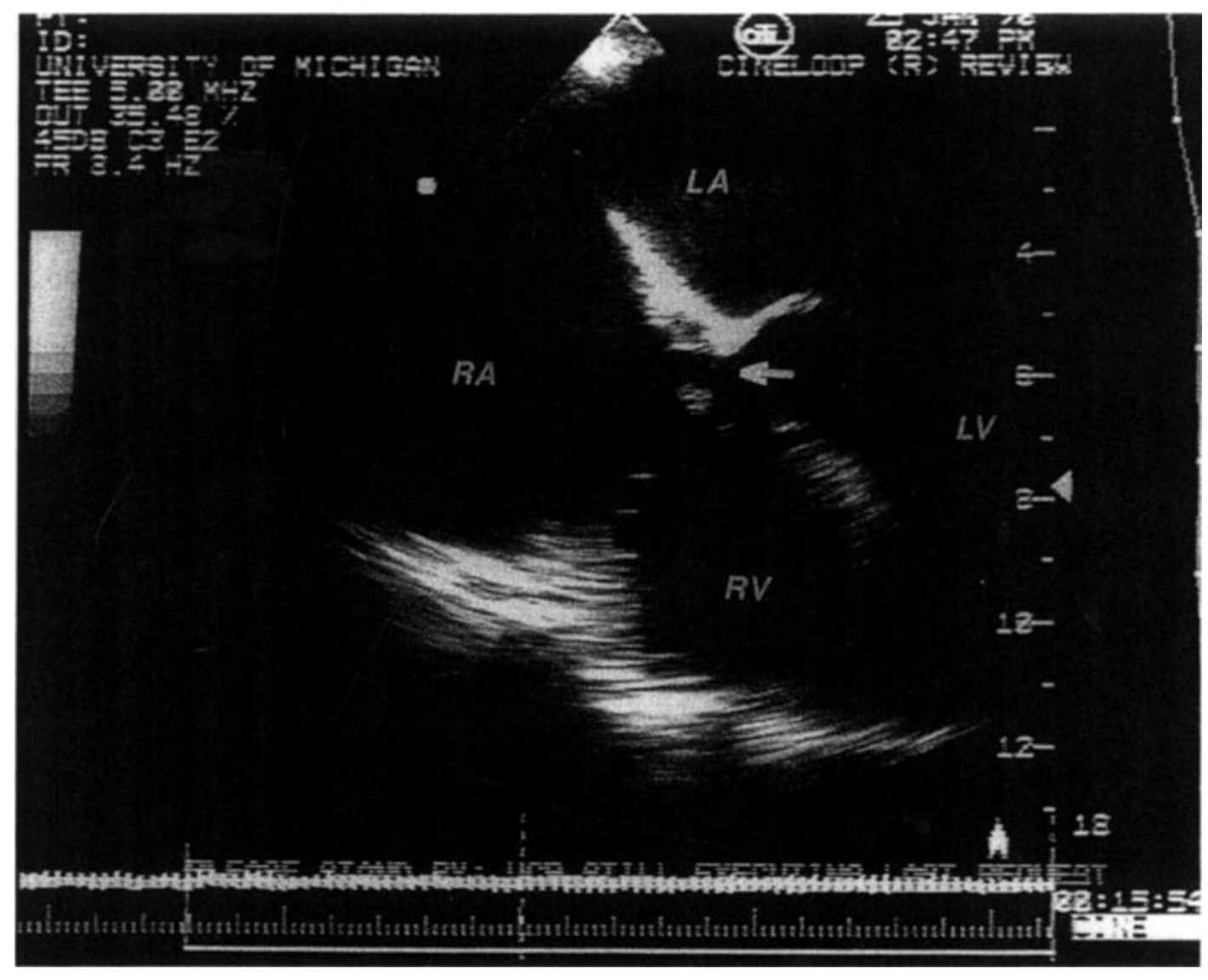

Figure 1. Transesophageal four-chamber echocardiographic view with direct visualization of the atrioventricular septal defect (arrow). The aortic valve is not in the imaging plane, thus confirming the location of the defect in the atrioventricular septum. LA = left atrium; $L V=$ left ventricle; $R A=$ right atrium; $R V=$ right ventricle. 


\section{TABLE I}

Hemodynamic Data From Cardiac Catheterization

\begin{tabular}{llc}
\hline \multicolumn{1}{c}{ Site } & \multicolumn{1}{c}{ Pressure } & \% Oxygen Saturation \\
\hline SVC & & 59 \\
High RA & & 65 \\
Mid RA & $13,20(11)$ & 75 \\
Low RA & & 68 \\
IVC & $42 / 7$ (RVEDP 6) & 68 \\
RV & $42 / 18(29)$ & 80 \\
PA & $17,16(13)$ & 84 \\
PCWP & $112 / 78(92)$ & - \\
Ao & $115 / 11$ (LVEDP 15) & - \\
LV & \\
\hline
\end{tabular}

Values separated by commas indicate $A$ and $V$ waves unless otherwise noted. Values in parentheses indicate mean pressure. $\mathrm{SVC}=$ superior vena cava; $\mathrm{RA}=$ right atrium; IVC $=$ inferior vena cava; $R V=$ right ventricle; $R V E D P=$ right ventricular end-diastolic pressure; $\mathrm{PA}=$ pulmonary artery; PCWP = pulmonary capillary wedge pressure; Ao $=$ aorta LV $=$ left ventricle; and LVEDP $=$ left ventricular end-diastolic pressure. pid regurgitation could not be excluded. Fluoroscopy revealed a paralyzed left hemidiaphragm. Coronary angiography was normal.

Six months after the accident, the patient underwent operation. After induction of anesthesia, a transesophageal echocardiogram was performed. In the four-chamber view from behind the left atrium, a $1-\mathrm{cm}$ defect in the atrioventricular septum could be easily visualized (Fig. 1). Color Doppler confirmed a left ventricular to right atrial shunt (Fig. 2). The base of the tricuspid valve was not involved in the defect and no significant tricuspid regurgitation was noted.

At the time of surgical exploration, the heart was encased in dense adhesions. A strong thrill was palpated over the right atrium and right ventricle. A right atriotomy was made, and the tricuspid valve was inspected and found to be

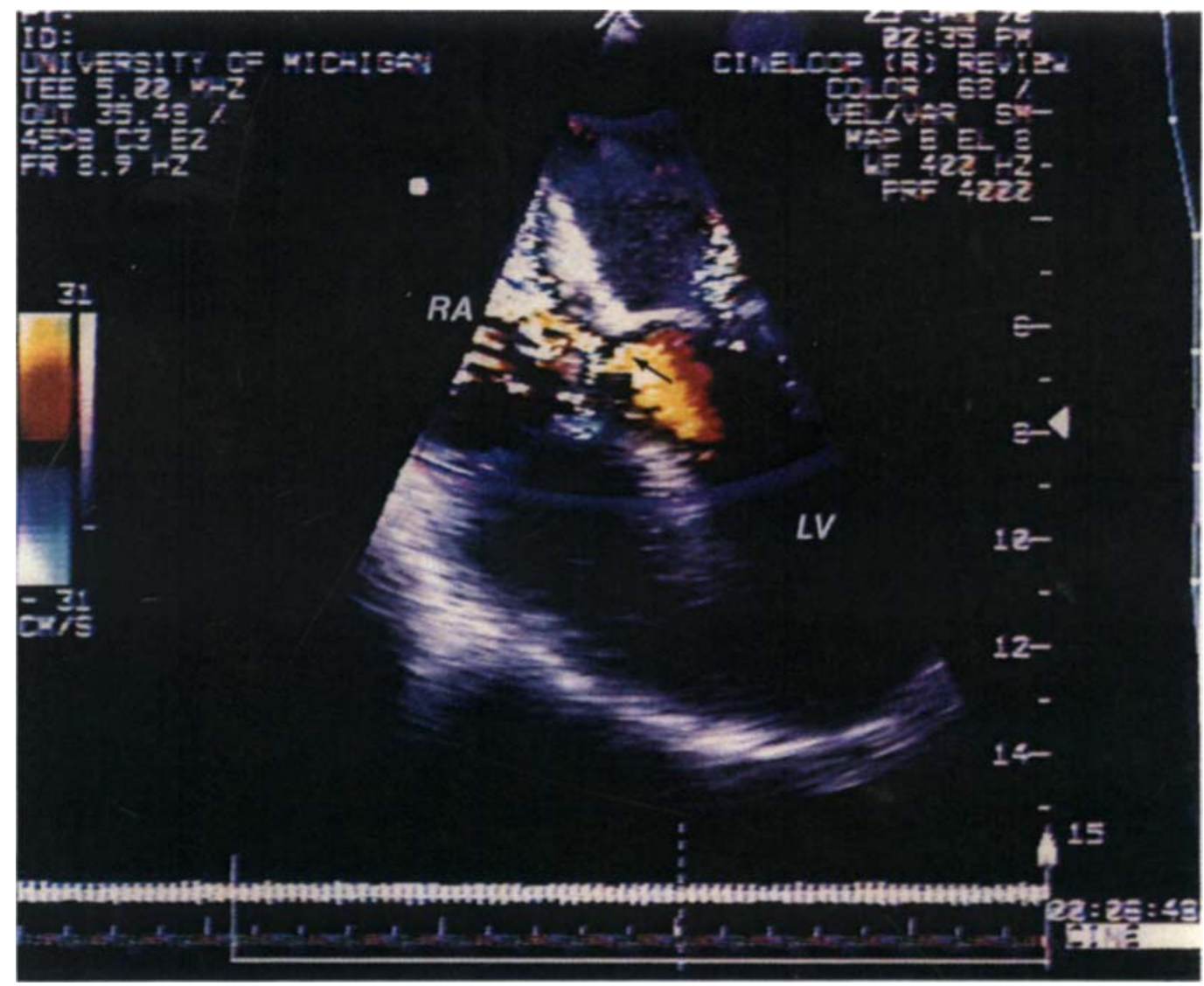

Figure 2. Color flow transesophageal four-chamber echocardiographic view demonstrating a high-velocity jet across the atrioventricular septal defect (arrow). LV = left ventricle; $R A=$ right atrium. 
normal. There was a left ventricular to right atrial septal defect approximately $1 \mathrm{~cm}$ in diameter just superior to the septal leaflet of the tricuspid valve. The edges of the defect were heavily scarred. The defect was closed with a Dacron patch. Following the procedure, a DDD pacemaker was inserted for complete heart block. He was discharged home and remains without cardiovascular symptoms.

\section{Discussion}

To our knowledge, this is the only patient in whom TEE was used to localize a left ventricular to right atrial septal defect. Distinguishing a left-to-right membranous ventricular septal defect from a left ventricular to right atrial septal defect can be difficult since both cause a holosystolic murmur. Demonstrating a right atrial oxygen saturation step-up at cardiac catheterization does not eliminate the possibility of a high membranous ventricular septal defect with tricuspid regurgitation or a concurrent atrial septal defect. Two out of the four previously reported cases of left ventricular to right atrial septal defects secondary to blunt thoracic trauma had tricuspid valve involvement. $^{2-5}$

As with the case presented here, three out of the four prior cases developed second- or thirddegree atrioventricular block, most likely secondary to anatomical proximity of the conduction system to the defect. In the three cases surviving past the first week, the atrioventricular block resolved, consistent with resolution of local edema and hemorrhage.
Only the two most recent cases were longterm survivors after undergoing successful surgical repair. Two cases died acutely, and a third died after failed recognition of this and an associated aortic valve defect. As with the case presented here, two cases followed a subacute clinical course with delayed rupture, presumably from liquefaction necrosis. Our case was unique in its markedly delayed recognition.

This patient represents a rare but potentially correctable consequence of blunt chest trauma. Transesophageal echocardiography allowed precise localization of the defect and was valuable in excluding other associated defects. Because of the degree of accuracy in establishing the diagnosis, transesophageal echocardiography should provide a more rapid diagnosis and may allow surgical repair in selected patients without pre-operative catheterization.

\section{References}

1. Parmley LF, Manion WC, Mattingly TW: Nonpenetrating traumatic injury of the heart. Circulation 1958;18:371.

2. Dunseth W, Ferguson TB: Acquired septal defect due to thoracic trauma. $J$ Trauma 1965;5:142.

3. Kanber GJ, Fort ML, Treger A, et al: Left ventricular-right atrial canal with aortic incompetence of probable traumatic origin. Am J Cardiol 1967;20:879.

4. Naccarelli GV, Haisty WK, Kahl FR: Left ventricular to right atrial defect and tricuspid insufficiency secondary to nonpenetrating cardiac trauma. J Trauma 1980;20:887.

5. Sims BA, Geddes JS: Traumatic heart block. $B r$ Heart J 1969;31:140. 